\title{
Long Period Superstructures in Close Packed Phases and Their Interpretation*
}

\author{
By Konrad Schubert**
}

\begin{abstract}
A survey is given on long period superstructures of close packed metallic phases comprehending shift variants of the $\mathrm{Cu}_{3} \mathrm{Au}$ type, stacking variants of the $\mathrm{Cu}_{3} \mathrm{Au}$ type and structures which display both variations. Shift variants and stacking variants obey valence electron rules for the shift density and h-density respectively. For the shift density a cut off interval exists in the neighbourhood of the valence electron concentration 1. The atom positions in the neighbourhood of the shift plane are somewhat changed and a possible rule for these changes is given. The shift normal is a direction of minimum elastic modulus following the presently known structures. Whole number half periods are preferred and give therefore a plateau of the shift density in dependence of the valence electron concentration. Nonintegral half periods are firmly established. There is a connection between the change of the axial ratio of the substructure and the change of the valence electron concentration. This axial ratio change is reduced by a second shift system. Shift variants occur with approximate valence electron concentrations 1 and 3 . The rule of minimum iteration is seldom violated in shift variants and in stacking variants. For all these facts interpretations considering the spatial correlation of the electrons are given. To confirm these interpretations several open problems which are enumerated must be solved. (Received September 29, 1972)
\end{abstract}

\section{Introduction}

The interest in long period crystal structures (LPS) runs parallel to the fundamental interest of all crystal structure research - the chemical binding in crystals. While the small elementary cells and the close packed structures occurring in many metallic phases suggested a short range binding in alloys the existence of long period superstructures in metallic close packed phases prooved that long range forces are of considerable influence. The study of long period superstructures gives therefore information on the properties of the long range forces in alloys. All experiments on LPS should be considered with respect to their significance to the long range forces. This is only possible by means of a model which brings many results on LPS produced by authors all over the world into a consistent picture. As is well known two different models for the interpretations of the LPS exist: (1) The band model of electron theory, which consideres the interaction between the Femisphere and the Brillouinplanes in the wave number space and (2) The spatial correlation model which leads to certain electrostatic fields (similar to the model of the ionic bond) the energy of which is minimized by the crystal structures. As the bandmodel, though it has been often used in the literature, is subject to limited application, it seems of interest to consider the spatial correlation model also. The fundaments of this model were theoretically formulated by $\mathrm{K}$. Husimi ${ }^{(1)}$. While the bandmodel is at present considerably elaborated, this cannot yet be maintained of the spatial correlation model. However, as a preliminary step to the development of the model its usefulness should first be established in the light of experiment. The fundamentals of the model $\operatorname{are}^{(2)}$; (1) The valence electrons form a lattice like spatial correlation of the range $50 \AA$ which

* Paper given at the 9th International Congress of Crystal. lography in Kyoto.

** Max Planck Institut für Metallforschung, Stuttgart, BRD.

(1) K. Husimi: Proc. Phys. Math. Soc. Japan, 22 (1940), 264. Trans. JIM minimizes the energy of the electron gas. (2) The interaction between valence electron correlation and crystal lattice finds an expression in commensurability relations between the two lattices and also in certain features of the structure (e.g. shifts, strains, electron rules).

\section{Shift Variants of the $\mathrm{Cu}_{3} \mathrm{Au}$ Type}

These stuctures have been found at first in the phases $\mathrm{TiAl}^{(3)}$ and $\mathrm{CuAu}^{(4)}$, they are nowadays extensively investigated and have even been made visible in the microscope ${ }^{(5)}$. A structure of the family is shown in Fig. 1, which explains also the concept of shift density $D_{s}=c_{F} / c$, where $c_{F}$ is the $c$ axis of the fundamental cell (substructure) and $c$ the $c$ axis of the

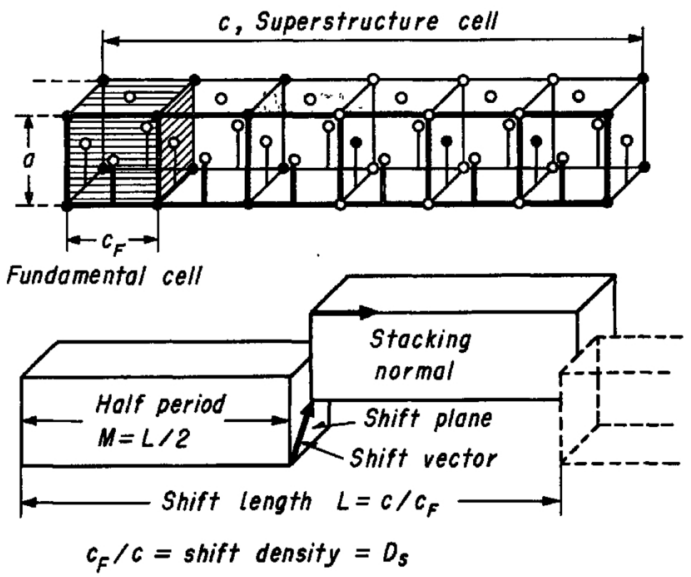

Fig. 1 A shift variant of the $\mathrm{Cu}_{3} \mathrm{Au}$ type and the concepts useful for its discussion.

(2) K. Schubert: Kristallstrukturen zweikomponentiger Phasen Springer Verlag, Berlin, (1964).

(3) W. L. Finkh, K. R. Horn and P. M. Budge: Trans. AIME Inst. Met. Div., (1931), p. 421.

(4) C. H. Johansson and J. O. Linde: Ann. Physik, 25 (1936), 1.

(5) S. Ogawa, D. Watanabé, H. Watanabé, and T. Komoda: Acta Cryst., 11 (1958), 872.

1973 Vol. 14 
real cell (superstructure). $c / c_{F}=L$ may be called shift length and $L / 2=M$ half period or domain thickness.

Several findings are collected in Fig. 2 and may be described as follows.

(1) A valence electron rule ${ }^{(6)}$ for the shift density was found by studying structures in ternary alloys and it may be expressed as $D_{s} \approx V-1$, where $V$ is the valence electron concentration. The rule has been amply confirmed ${ }^{(7)}$ and was interpreted by the bandmodel $^{(7)}$ and also by the spatial correlation of valence electrons ${ }^{(2)(6)}$. In the spatial correlation interpretation because of $2=l_{c E}-l_{c A}$ where $l_{c E}=$ number of electron layers per $c$ length and $l_{c A}=$ number of atom layers per $c$ length an array of electrostatic dipole moments is built up the energy of which is minimized by the shifts Fig. 3 .

(2) A correlation between the increment $c_{F} / a-1$ and the shift density states that both have the same sign. This may be considered also as a correlation between $c_{F} / a-1$ and $V-1$.

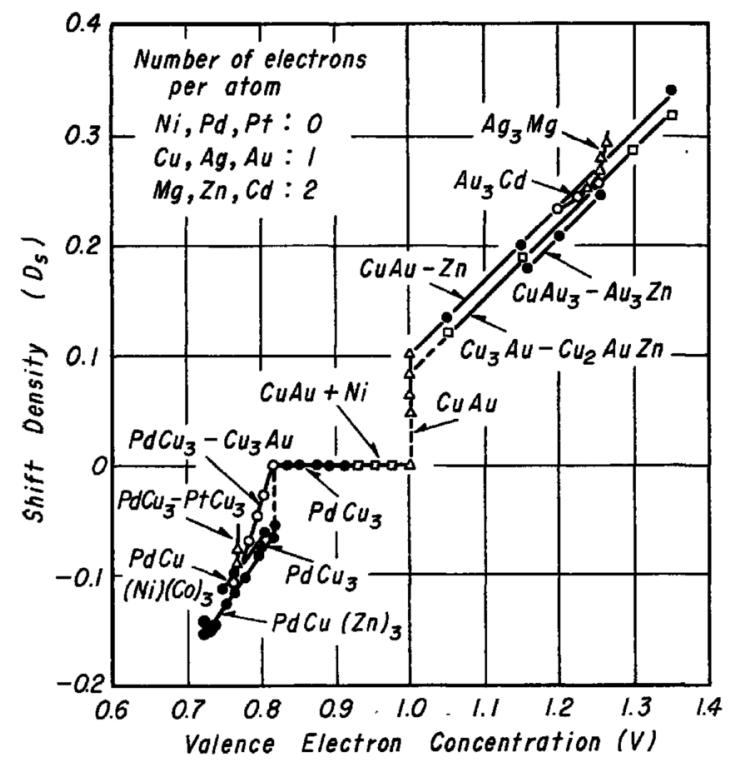

Fig. 2 The dependence of shift density on valence electron concentration.

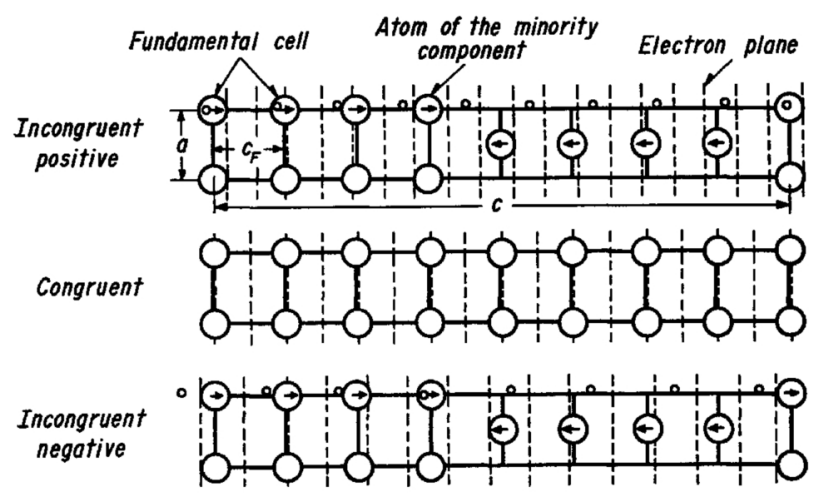

Fig. 3 Commensurability of A1 correlation (which is congruent in the $a \times a$ plane) in the $c$ direction.

(6) K. Schubert, B. Kiefer, M. Wilkens and R. Haufler: Z. Metallk., 46 (1955), 692.

(7) H. Sato and R. S. Toth: Long Period Superlattices in Alloys, Metallurgical Society Conf., Vol. 29 (1965) 295. . . 419 (further references in this paper).
(3) The cut off interval near $V-1$ characterizes the range of $50 \AA$ of the valence electron correlation. Because of the zero point energy the range should also exist at temperature $T=0 \mathrm{~K}$, while in the band model interpretation the interval should disappear. Because of slow diffusion this cannot be verified. The range has been corroborated by other methods ${ }^{(8)}$.

(4) A depression of the effective valence electron concentration with increasing temperature has been measured $^{(9)}$. It may be interpreted by the fact that at higher temperatures valence electrons populate interstitial places of the correlation, thus diminishing the effective valence electron concentration. This may be overcompensated by a component having a temperature dependent valence electron contribution.

(5) The $\mathrm{Au}$ atom has a temperature dependent valence electron contribution as is understood from the fact that $\mathrm{CuAuI}$ has a structure without shifts, while at higher temperatures CuAuII has a shift structure.

(6) The temperature dependent valence electron contribution of $\mathrm{Au}$ is influenced by the valence electron concentration of the alloy: at higher valence electron concentrations $\mathrm{Au}$ has the valence electron contribution 1.

(7) An increase of the nominal valence electron concentration to an effective valence electron concentration is caused by the fact that atomic cores occupy valence electron places.

Further features of shift variants of $\mathrm{Cu}_{3} \mathrm{Au}$ will be considered in the next paragraphs.

\section{Atom Displacements in Shift Variants of the $\mathrm{Cu}_{3} \mathrm{Au}$ Type}

As the atom arrangement in the neighbourhood of the shift plane is different from that in regions mostly remote from shift planes, certain displacements of atoms away from the "rideal" site are to be expected. These displacements may be considered as a density modulation of the structure, which causes satellite reflexions at the fundamental structure reflexions, especially visible at the wave-number point $(h, k, l)=$ $(0,0,0)^{(10)}$. Another simple model consists in the assumption that the elementary cells of one domain have a lattice constant which is a little shorter (longer) than $c / L$; this results in a dilatation (contraction) of the atom distances near the shift plane and the intensity of a superstructure dumpbell becomes asymmetrical: the intensity of the dumpbell component with larger (smaller) $\theta$ is higher than the other ${ }^{(11)}$. A more detailed analysis ${ }^{(12) \sim(14)}$ permits a determination of all

(8) H. Gleiter: Acta Met., 16 (1968), 1067.

(9) S. Ogawa, A. Terada and H. Iwasaki: Ninth Int. Congr. Cryst. Coll. Abstr., VII-5, (1972).

(10) S. Ogawa and D. Watanabé: Acta Cryst., 7 (1954), 377.

(11) M. Wilkens and K. Schubert: Z. Metallk., 48 (1957), 550.

(12) H. Iwasaki, M. Hirabayashi and S. Ogawa: J. Phys. Soc. Japan, 20 (1965), 89.

(13) K. Okamura, H. Iwasaki and S. Ogawa: J. Phys. Soc. Japan, 24 (1968), 569.

(14) K. Okamura: J. Phys. Soc. Japan, 28 (1970), 1005. 


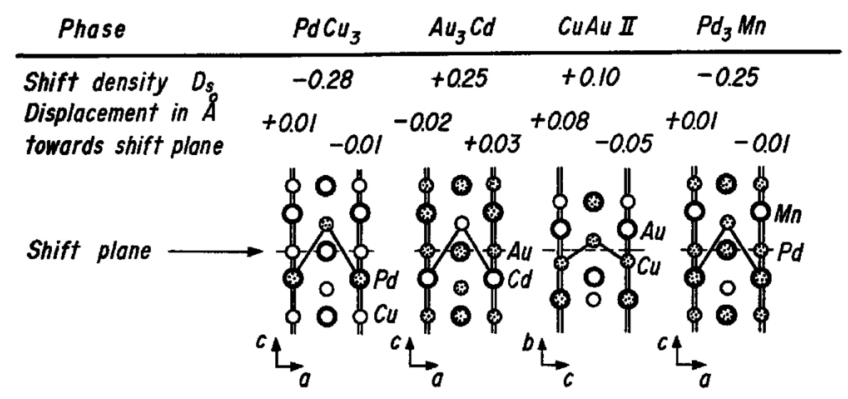

Fig. 4 Small displacements of atoms near the shift plane (following H. Iwasaki, K. Okamura and S. Ogawa, 1971).

atom displacements in the cell Fig. 4. The findings may be summarized to the effect that the heavier component moves toward (away from) the shift plane when the axial ratio is diminished (increased) ${ }^{(11)(14)}$ as compared with the cubic structure. As the heavier atom is in the cases studied till now the one with less valence electrons, we have the tentative rule that the component with more electrons moves contrary to the change of $c / a$. This is compatible with the valence electron correlation interpretation: The strained (compressed) correlation tends to remove (near) the supporting atoms from the shift plane. An influence of the nearest neighbour interactions ${ }^{(15)}$ is also present, but one would anticipate that the heavier atom (which interacts stronger) is always moved to the shift plane whereas $\mathrm{Au}$ in $\mathrm{Au}_{3} \mathrm{Cd}$ moves away from the shift plane. On the other hand, if it where only a radius problem the bigger atom should move away from the shift plane, whereas $\mathrm{Cd}$ in $\mathrm{Au}_{3} \mathrm{Cd}$ moves to the shift plane. More research seems necessary for the firm establishment of that tentative rule.

A further fact has been found by the quantitative structure determination ${ }^{(14)(16)(17)}$ : in the reighbourhood of the shift plane there is a certain disordering of the components occupying an atomic site. This is compatible with the spatial correlation interpretations: In the middle of the domain the atoms bear strong and parallel electrostatic dipol moments in the direction of the shift normal, while at the border of the domain (shift plane) the dipol moment is weak and antiparallel with the moment of one of the neighbouring layers parallel to the shift plane. We may therefore understand that the atoms far from the shift plane are held more close in their superstructure positions. The phase ${ }^{(16)} \mathrm{Pd}_{3} \mathrm{Mn}$, which has $(c / a)_{F}=0.990$ and $L=4$ is to be compared with $\mathrm{PdCu}_{3}$, as neutron diffraction showed 2. 9 valence electrons for $\mathrm{Mn}$ and 0 for $\mathrm{Pd}^{(18)}$.

\section{Non-integral and Integral Half Periods}

A quasi continuous dependence of the shift density $D_{s}$ on the valence electron concentration is experimentally firmly established. As in the atomic model only whole number half periods are possible, a crystal with non-integral half period must contain a mixture of the two neighbouring whole number half periods $M^{\prime}$ and $M^{\prime \prime}$. Fujiwara ${ }^{(19)}$ observed, that on a "dumpbell series" $h k l$ with $h=$ const., $k=$ const., $h+k=$ $2 n+1$ the amplitude is given by $F=\Sigma_{n}^{0 \ldots N-1} f_{L}(n)$ $\exp 2 \pi i n l$ where $f_{L}(z)=1$ (respectively -1 ) for $L m \leqq z<L(m+1 / 2) \quad$ (respectively $L(m+1 / 2) \leqq$ $z<L(m+1))$ where $m=0,1,2,3, \ldots$ If this is extended to noninteger $L / 2$ an array of domains of different thickness is produced which gives ${ }^{(19)(20)}$ the dumpbell reflections as sharp maxima. From Fig. 5 it is easily understood that the dipole array of the spatial correlation interpretation and Fujiwara's antiphase factor are mathematically equivalent: the positive value of $f$ corresponds to positive dipole moments and negative $f$ to negative moments. Therefore the dipole array is the physical reason for the observed domain array, which was called "uniform" by Fujiwara and which may be characterized by a maximum $\left(M^{\prime}\right.$, $\left.M^{\prime \prime}\right)$ - correlation (intercorrelation), or minimum $\left(M^{\prime}\right.$,

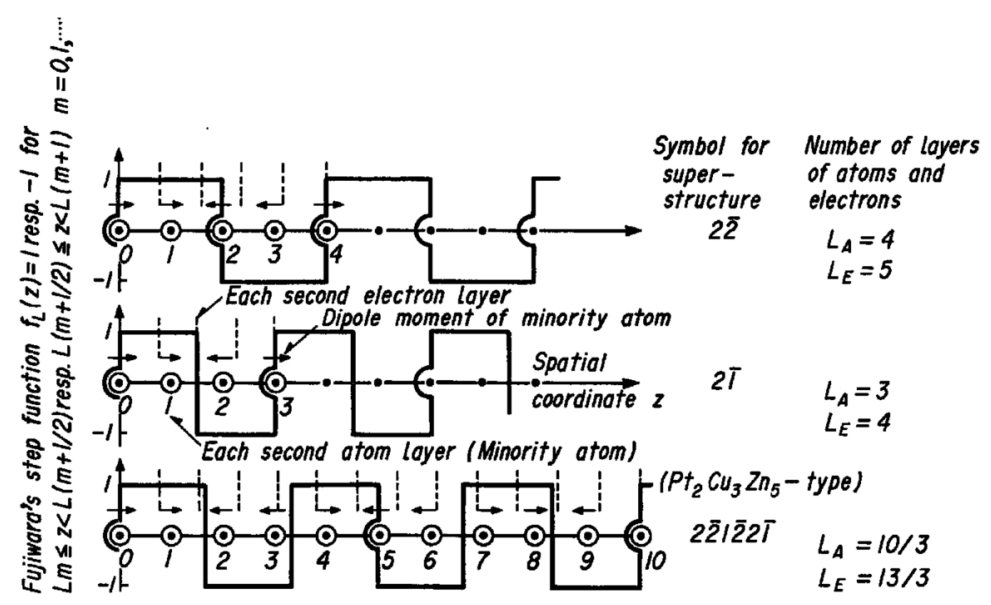

Fig. 5 Commensurability of the valence electron correlation in the direction of the shift normal and Fujiwara's step function.

(15) M. Tachiki: Phys. Rev., 150 (1966), 440.

(16) A. Gangulee and S. C. Moss: J. Appl. Cryst., 1 (1968), 61.

(17) H. Iwasaki, K. Okamura and S. Ogawa: J. Phys, Soc. Japan, 31 (1971), 497.
(18) H. Krén and G. Kádár: Phys. Letters, 29A (1969), 340.

(19) K. Fujiwara: J. Phys. Soc. Japan, 12 (1957), 7. 
$\left.M^{\prime}\right)$ - and $\left(M^{\prime \prime}, M^{\prime \prime}\right)$-correlation (selfcorrelation). They may therefore be named distributions of minimum iteration.

If $L$ is a fairly simple faction of integers, a fairly small supercell is produced by the denominator of the fraction. Additional superstructure maxima were calculated by Fujiwara for this case and were in fact experimentally found ${ }^{(21) \sim(23)}$. When $L$ changes, say by $1 \%$, because of a fluctuation of the valence electron concentration the big superstructure cell is heavily changed; the main intensity dumpbell maxima remain the same, while the additional maxima change. Therefore the additional maxima are generally not observed.

It is not so that integral and nonintegral half periods are energetically equivalent. The linear dependence of $D_{s}$ on $V$ is only a first approximation ${ }^{(11)(22)}$, at certain $D_{s}$ values the valence electron concentration may change in some measure without change $D_{s}$. This is not unexpected, as the commensurability between valence electron correlation and crystal lattice gives energetic preferences for certain shift densities while other parameters leading to the concept of effective valence electron concentration allow a change of $V$ at constant $D_{s}$. This problem was considered in some detail by Tachiki and Maekawa ${ }^{(24)}$, and their theory is compatible with the spatial correlation interpretation because of the commensurability rule.

\section{The Axial Ratio $c / a$ of the Fundamental Cell}

Agreeing with the assumption that the excess or defect of the valence electron concentration from the value 1 leads to the shift phenomenon, there remains the question why there is at all a tetragonal deformation of the fundamental cell while the bandmodelenergy would be gained by the split of the reflections alone (caused by the shifting). In the spatial correlation interpretation electron planes must be inserted normally to a given direction while the commensurability is preserved in the directions vertical to the given direction. This raises a problem of elasticity in the system consisting of the atom correlation (crystal lattice) and the valence electron correlation (electron lattice). As the two correlations are at $V=1$ congruent, they must distort one another in the same direction with opposite sign at $V>1$ or $V<1$; the energy of the distortion will be least, when the direction has mimimum elastic modulus. As is well known in Cu-type lattices [001] is the direction of minimum elastic modulus. Thus the shift normal [001] is compatible with elastic data. Comparing the strain of the

(20) J. Kakinoki and T. Minagawa: Acta Cryst., A28 (1972), $120,308$.

(21) K. Fujiwara, M. Hirabayashi, D. Watanabé and S. Ogawa: J. Phys. Soc. Japan, 13 (1958), 167.

(22) Y. Khan, B. V. R. Murty and K. Schubert: J. LessCommon Metals, 21 (1970), 293.

(23) W. Gaudig and H. Warlimont: Z. Metallk., 60 (1969), 488.

(24) M. Tachiki and S. Maekawa: J. Phys. Soc. Japan, 28 (1970), 375.

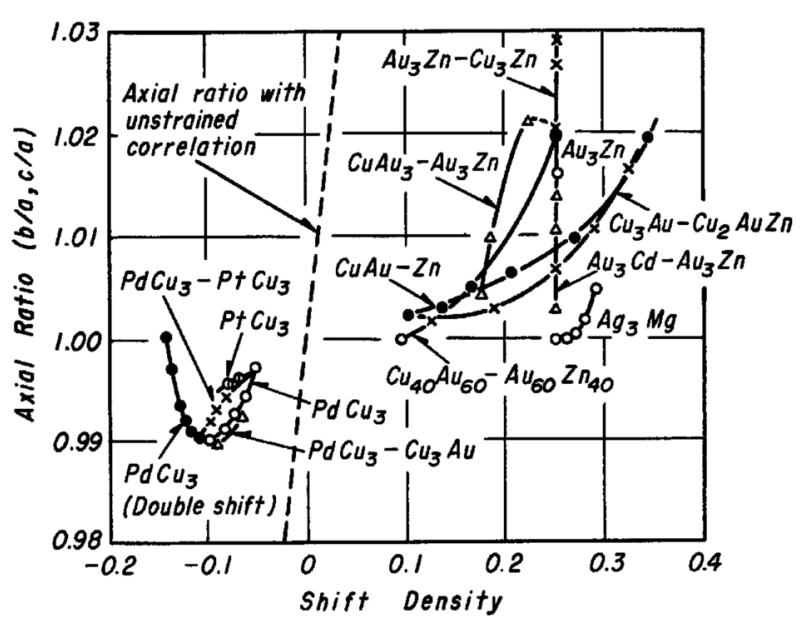

Fig. 6 Observed axial ratio of the fundamental cell in dependence of the shift density.

two correlations (Fig. 6) one finds that the crystal is by a factor of $5 \ldots 10$ more rigid than the valence electron gas. An apparent exception is the structure of the element Indium; this observation leads to an analysis of the core electron correlation of brass like phases $^{(25)}$.

A quite remarkable dependence of strain on concentration is shown by the double shift structures, the first of which was analysed by Watanabe, Hirabayashi and Ogawa ${ }^{(26)}$. It seems that a stoichiometric deviation from the ideal composition is necessary for the stabilisation of a double shift structure. In the case of $\mathrm{PdCu}_{3}$ the additional $\mathrm{Pd}$ atoms accumulate in planes parallel to the shift normal so that the Al correlation is cut in layers parallel to the shift normal. Because of the cross strain dipol moments are induced vertically to the shift normal so that a new shift system becomes stable, and at the same time the stress in direction of the shift normal is reduced so that $c_{F} / a$ goes back to 1 . The shift density increases with increasing Pd content. Further research seems desirable to extend the knowledge on double shift structures.

\section{Shift Structures in Non Brass Like Phases}

An alloy is called brass like if the core is at its surface of the filled $d$ shell type and the valence electron concentration is not too high. A set of phases characterized by $\mathrm{VNi}_{3}$ is no more brass like, but shows shift variants of the $\mathrm{Cu}_{3} \mathrm{Au}$ type. We must consider the 5 outer electrons of $V$ as valence electrons and also the 10 outer electrons of $\mathrm{Ni}$ as core electrons, in order to get a brass like valence electron concentration and the valence electron correlation ${ }^{(27)} a / \sqrt{2}=a_{\mathrm{A} 2} l_{c}=2 \times$ $2.9 \approx 6$, which means that the vektor $\left(a_{1}+a_{2}\right) / 2$ is equal to the vector $a_{\mathrm{A} 2}$ of an $\mathrm{A} 2$ correlation and the number of electron layers per $\mathrm{c}$ axis is $2 \times 2.9$ assuming that the correlation is unstrained and 6 assuming

(25) K. Schubert: Recent developments in metallurgical Sci. and Tech., Indian Inst. Met., New Delhi, (1972), p. 485.

(26) D. Watanabé, M. Hirabayashi and S. Ogawa: J. Phys. Soc. Japan, 11 (1956), 226; 12 (1957), 259.

(27) K. Schubert: Bull. Soc. fr. Min. Crist., 91 (1968), 575. 
that it is slightly strained. This proposal explains not only the axial ratio $2 \times 1.02$ of the cell but also the structural shift with density $D_{s}=1 / 2$. The assumption that the 5 outer electrons of a $V$ atom are quasiunformly distributed in the crystal is proved by the structure of $\mathrm{TiPt}_{\mathbf{8}}$, which is a tetragonal superstructure of the $\mathrm{Cu}$ type with $a=8.3 c=3.9 \AA$, and which permits the correlation $^{(28)} a / 2=a_{\mathrm{A} 2} l_{c}=2$. The fact, that the shift density $1 / 2$ does not occur in brass like (undistorted) phases must be interpreted by the assumption that the slightly different core electron correlation in $\mathrm{VNi}_{3}$ favours the shift length 2 . The phase $\mathrm{VNi}_{2}$ which is in equilibrium with $\mathrm{VNi}_{3}$ has a $\mathrm{MoPt}_{2}$ structure, which is an orthorhombic superstructure of the $\mathrm{Cu}$ type $(a=2.56 \quad b=7.64 \quad c=3.55 \AA)$. Because of the strong change in crystal structure a strongly changed valence electron correlation is to be expected: $a=d_{\mathrm{B} 1} \sqrt{2} \quad b=3 d_{\mathrm{B} 1} \sqrt{2} \quad c=2 d_{\mathrm{B} 1}$ which does not necessitate shifting with shift normal $c$ and which favours the $V$-chains along the lattice vector $a$ by dipole moment formation.

A further chemical branch of the shift variants of $\mathrm{Cu}_{3} \mathrm{Au}$ is given by the phase $\mathrm{TiAl}_{3}$. Though the valence electron concentration 3.25 is quite different from that in the brass like shift structures, the physical explanation of the shifts remains the same. Counting $4 \mathrm{Ti}$ electrons and $3 \times 3 \mathrm{Al}$-electrons in the cell one gets the valence electron correlation $a / \sqrt{2}=a_{\mathrm{A} 1} l_{c}=$ $6.3 \approx 6.5$, where the latter number gives precisely the number of valence electrons in the cell. The change of commensurability between $\mathrm{VNi}_{3}$ and $\mathrm{TiAl}_{3}$ is an instructive explanation of the phenomenon of "come back" of a structural type, when the valence electron concentration increases. The electron layer number $l_{c}=6$ gives exactly the $\mathrm{TiAl}_{3}$ structure if we count only 3 of them. As the superstructure lines of carefully annealed $\mathrm{TiAl}_{3}$ have precisely the $\theta$ values of the $l_{c}=6$ structure. It must be concluded that $l_{c}=7$, and that each electron plane contributes to the dipole moments. The exial ratio of $\mathrm{TiAl}_{3}$ gives much less compression of the valence electron correlation than in brass like alloys reflecting the weaker core electron influence; Fig. 7 shows $^{(30)}$ that this is the case also for a comprehensive class of related phases.

The fact that $\mathrm{VAl}_{3}$ is isotypical to $\mathrm{TiAl}_{3}$ is an example for a curious phenomenon: in the neighbourhood of the $V$ atom the Al correlation may be assumed to be filled by one outer electron to a B1 correlation. This is a slight violation of Hunds' rule, as it has to be assumed that the filling electron has another spin than that of the electrons in the Al correlation; the correlative forces compel the fifth electron to change spin instead of the sixth as in the free atom.

Because of the changed commensurability in the tetragonal basal plane one has now to count in deriving the dipole moments at the minority component

(28) P. Krautwasser, S. Bhan and K. Schubert: Z. Metallk., 59 (1968), 724.

(29) K. Schubert, P. Esslinger et al.: Naturwiss., 43 (1956), 248.

(30) A. Raman and K. Schubert: Z. Metallk., 56 (1965), 99.

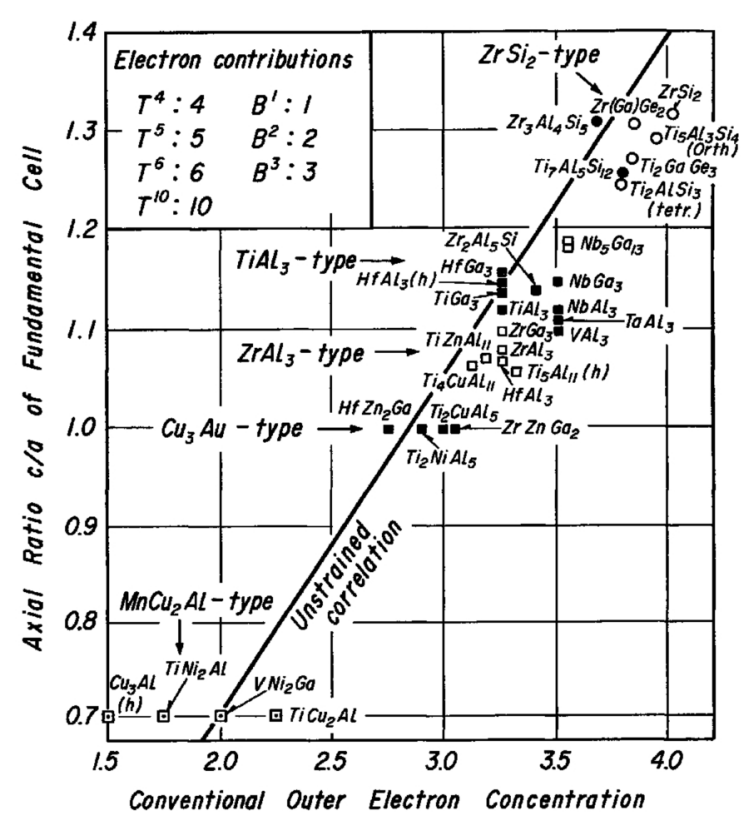

Fig. 7 Axial ratio of shift variants of the $\mathrm{Cu}_{3} \mathrm{Au}$ type in $\mathrm{T}-\mathrm{B}$ phases.
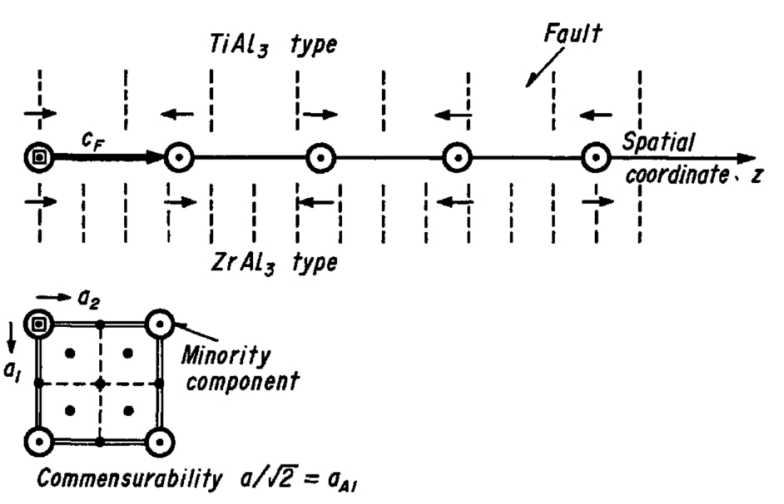

Fig. 8 Commensurability of the valence electron correlation and dipole array, in the $\mathrm{T}-\mathrm{B}$ phases $\mathrm{TiAl}_{3}$ and $\mathrm{ZrAl}_{3}$.

no more each second electron layer but in fact each electron layer. The increased density of places would favour to count each layer. In the latter case the $\mathrm{ZrAl}_{3}$ structure is favoured assuming 13 electron layers per c axis Fig. 8. A similar case has been found in $\mathrm{Pd}_{3} \mathrm{Tl}$ and $\mathrm{Pd}_{3} \mathrm{In}$, where the (h) phase is of the $\mathrm{TiAl}_{3}$-and the (r) phase of the $\mathrm{ZrAl}_{3}$-type; the correlation is $a=a_{\mathrm{A} 1}$ $l_{c} \approx 1.5$; when only each second electron layer is effective the (r) structure is favoured, when each electron layer is effective, the (h) structure is favoured.

More complicated shift structures are ${ }^{(2)}$ for instance $\mathrm{ZrSi}_{2}, \mathrm{ZrGa}_{2}, \mathrm{HfGa}_{2}, \mathrm{Nb}_{5} \mathrm{Ga}_{13}$.

\section{Further Structural Variants}

An important family of structures related to the shift variants of the $\mathrm{Cu}_{3} \mathrm{Au}$ type are the internally distorted structures. As examples $\mathrm{Au}_{3} \mathrm{Zn}(\mathrm{r})$ and $\mathrm{Pt}_{5} \mathrm{Ga}_{3}, \mathrm{Pt}_{3} \mathrm{Ge}$ may be named. To bring these structures into a consistent picture an analysis of the outer core electron correlation is necessary. Therefore they shall not be considered here in connection with the more symmetrical LPS structures. 
A structural family which is in a quite remarkable relation to the variants of the $\mathrm{Cu}$ type are the beta Hume-Rothery phases of the $\mathrm{CsCl}$ type. Starting from $\mathrm{Cu}$ with a valence electron correlation which may be described by $a=a_{\mathrm{A} 1}$ the substitution of $\mathrm{Zn}$ for $\mathrm{Cu}$ may result in a shift variant with a tetragonally strained substructure cell. (Actually the difference of the atomic dipole moments is so weak, that the cubic disordered modification persists down to temperatures where sensible diffusion ceases.) Further substitution of $\mathrm{Zn}$ results in the stabilisation of the $\mathrm{CuZn}$ structure, which is strongly tetragonally contracted as compared with the $\mathrm{Cu}$ structure. This may be explained by the assumption that in the tetragonal basis plane the commensurability of the valence electron correlation with the $\mathrm{Cu}$ type structure has changed from $a=a_{\mathrm{A} 1}$ to $a / \sqrt{2}=a_{\mathrm{A} 1}$ i.e. by a rotation about $45^{\circ}$ and contraction. In the $\mathrm{CuZn}$ structure one of the places of the A1 correlation is occupied by an atom, so that the Hume-Rothery rule $V=3 / 2$ is valid. Increase in $V$ results in Bradley vacancy formation following the Norbury rule and in many types of related crystal structures ${ }^{(2)}$. Curiously enough there is another branch of the $\mathrm{CsCl}$ type, a representative of which is $\mathrm{Pd}_{40} \mathrm{Cu}_{60}(\mathrm{r})$; it has ${ }^{(2)}$ the valence electron correlation $a=d_{\mathrm{A} 1}$, where $d_{\mathrm{A} 1}$ is the distance of the $\mathrm{A} 1$ type correlation, and the number $l_{c}$ of electron nets parallel to the basal plane is 1.4 ; this correlation is twinned, so that the crystal remains cubic. A bandmodel interpretation of this phase is not known.

\section{Hexagonal Close Packed Structures with Stacking Variance}

While in the phase $\mathrm{VNi}_{3}$ we have a shift variant of shift density $1 / 2$, such structures are generally not found in truly brass like structures. In brass like alloys another commensurability becomes more stable, as the deformations of the $\mathrm{Cu}_{3} \mathrm{Au}$ shift variants at $V \approx$ 1.3 become large, so that the elastic modulus law is no more valid. The new commensurability may be assumed as congruent in the (111) plane of the $\mathrm{Cu}$ type which is now called the basal plane; the type of the correlation may be an $\mathrm{Al}$ or a $\mathrm{Cll}$ correlation both of which have a hexagonal plane; we say then that the correlation is stacked in an Al or C11 manner. Increase of valence electron concentration results in inserting more electron planes parallel to the basal plane. This fact leads to stacking variations of the close packed layers ${ }^{(31)(32)}$ of the $\mathrm{Cu}$ type, which may be described by $\mathrm{ABCB}$ or by the first derivative ++or by the second derivative $c h$ where $c$ means the "cubic" stacking and $h$ the "hexagonal" stacking. The $h$ stacking is comparable to the shifting and it is gratifying that a valence electron rule exists which gives the $h$-density $D_{h}$ in dependence of $V$ as $D_{h} / 2=$ $V-1$ (Fig. 9). Considering more closely the structures

(31) J. Wegst and K. Schubert: Z. Metallk., 49 (1958), 533.

(32) B. C. Giessen and N. J. Grant: Acta Cryst., 17 (1964), $615 ; 18$ (1965), 1080

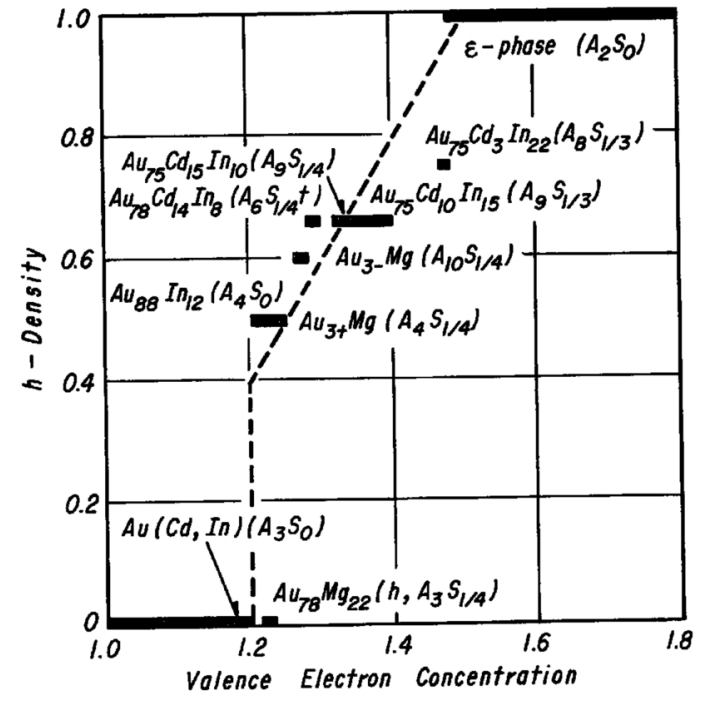

Fig. 9 Dependence of the $h$ density on valence electron concentration.

one finds that the distribution of minimum iteration is most frequently fulfilled. (Exceptions to this rule must be the object of a later more refined theory). This makes the interpretation probable that the stacking variants are also caused by arrays of electrostatic dipole moments. Transforming this to $2\left(l_{c E}-l_{c A}\right)=$ $D_{h} l_{c A}$, the left side may be understood as number of changes of direction of electric dipole moment per cell and the right side as number of $h$-stackings. The dipole moments may be assumed as in Fig. 10. The vertical of the little triangles designates an atomic site; the top pointing left or right designates the direction of the dipole moment ${ }^{(27)}$. The empirical rule that the repetition ${ }^{(2)}$ of the dipole direction gives a $c$-stacking while the change of the direction gives an $h$-stacking may be interpreted in the following way. If layer A and layer B bear a positive dipole moment (pointing upwards) they have a positive electrostatic charge on thier upper site and a negative on their bottom site; as the negative charges of $\mathrm{B}$ are shifted towards an A site, a third layer becomes $\mathrm{C}$ or $\mathrm{A}$ respectively if it bears a positive or negative dipole moment. This model gives the sequence of layers per cell of $6,4,10,9,8,21$ and 2 with increasing valence electron concentration. This sequence has been confirmed $^{(27)(33)}$ in close packed structures (Fig. 11). Also in phases of the $\mathrm{Cu}_{2} \mathrm{Mg}$ family the sequence has been found by Komura and coworkers ${ }^{(34)}$. These structures may be designated as double replacement structures as one $\mathrm{Mg}$ replaces two $\mathrm{Cu}$ in a close packed structure of $\mathrm{Cu}$. By the double replacement two layers of the close packing become a structural unit (Komura calls it "quadruple layer"). Disregarding each second electron layer we regain the rule of Fig. 10. In order to compare the valence electron concentration $l_{c E} / l_{c A}$ of Fig.10 with the valence electron concentrations of the members of the $\mathrm{Cu}_{2} \mathrm{Mg}$ family,

(33) H. U. Pfeifer, S. Bhan and K. Schubert: J. Less-Common Metals, 14 (1968), 291.

(34) Y. Komura, M. Mitarai, A. Nakane and S. Tsujimoto: Acta Cryst., B28 (1972), 976. 


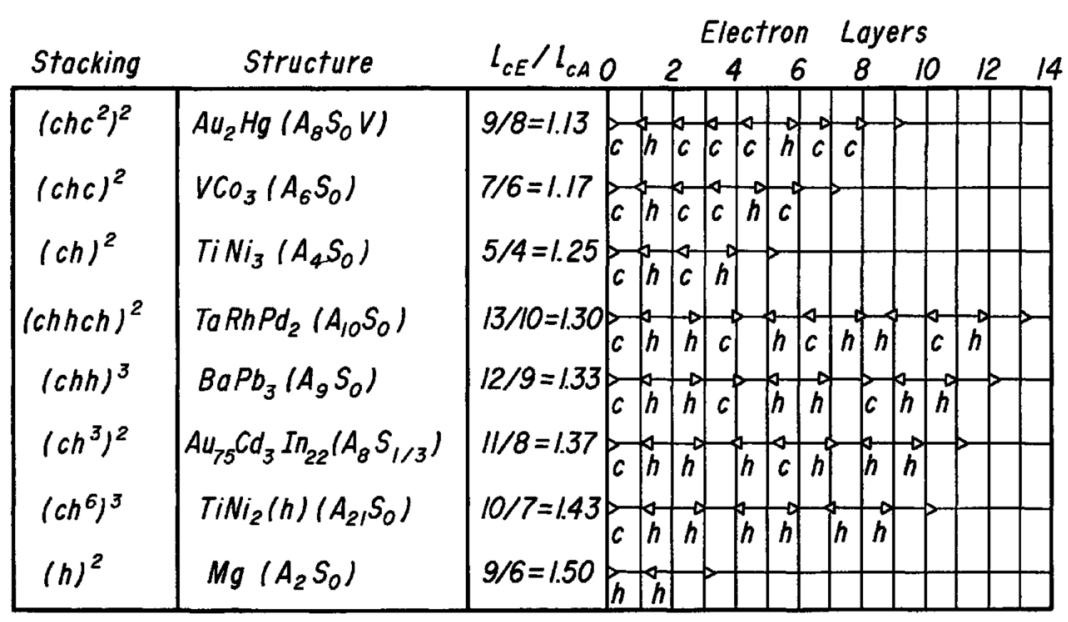

Fig. 10 Dipole array in stacking variants of close packed structures.

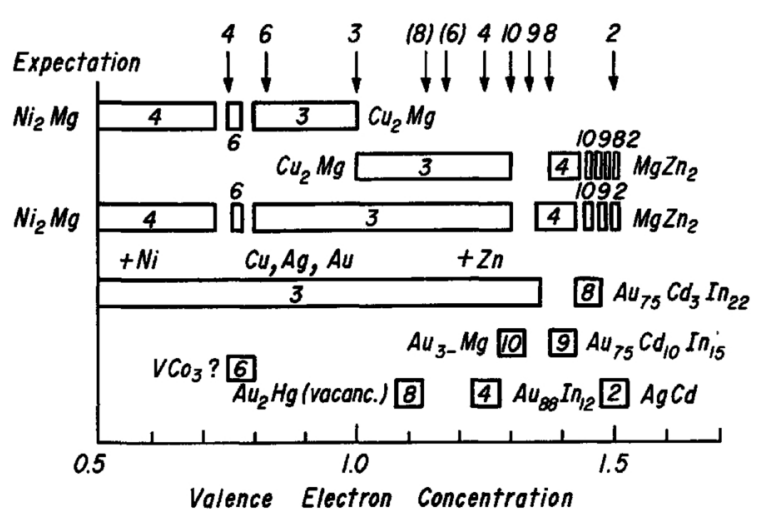

Fig. 11 Number of layers per cell in close packed structures; $\mathrm{Cu}_{2} \mathrm{Mg}$ family following $\mathrm{Y}$. Kamura, with transformed VEC.

the latter have to be multiplied by the factor $3 / 4$ (cf. ${ }^{(2)}$ p. 162). All stacking variants of the $\mathrm{Cu}_{2} \mathrm{Mg}$ family obey the rule of mimimum iteration.

\section{Hexagonal or Quasihexagonal Stacking Variants with Further Changes}

In fact the hexagonal stacking variants with the small basal plane are the exceptional cases. This is not surprising as a strain of the spatial correlation of electrons in only one direction is improbale. The following variants have been observed.

(1) $\mathrm{A} \mathrm{Mg}$ type close packed structure shows in the basal plane a simple shift structure. Example $\mathrm{Cu}_{3} \mathrm{Sn}$.

(2) A close packed stacking variant shows in the basal plane a shift of the superstructure. Example $\mathrm{Au}_{3}-\mathrm{Mg}$ in Fig. 12.

(3) A Mg type structure which shows a two dimensional superstructure in the basal plane. Example $\mathrm{Cu}_{10} \mathrm{Sb}_{3}(h)^{(2)}$.

(4) A close packed stacking variant shows a twodimensional superstructure in the basal plane. Example: $\mathrm{Au}_{77} \mathrm{Mg}_{23}$. Further structural types of this family have been analysed by Hirabayashi, Sato and their coworkers $^{(35)}$.

(35) M. Hirabayashi, S. Yamaguchi, N. Ino, H. Sato and R. S. Toth: J. Phys. Chem. Solids, 31 (1970), 77.

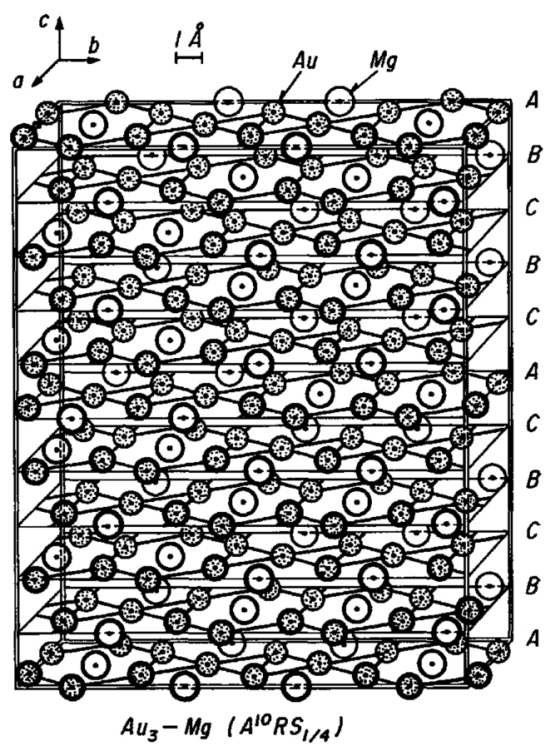

Fig. 12 Crystal structure of $\mathrm{Au}_{3}-\mathrm{Mg}$ a 10 layer stacking variant with shift density $1 / 4$ in the basal $a \times b$ plane.

\section{Concluding Remarks}

The number of variants of structures which belong to the closest packings of equal spheres has increased beyond expectation and further new structure types will be analysed in the future. However, it can be anticipated that the number of structure types of this family is finite, so that the time will come where all variants are known; this may be concluded from the finite number of chemical elements and from the finite range of the binding forces. Nevertheless we cannot at present pretend that we understand satisfactorily this structure family. In my view the following tasks stand before us and should be attacked:

(1) Analysis of further structures belonging to the above family. Investigation of structural details such as little atom shifts and the like.

(2) Examination whether the bandmodel in its present form permits to explain the facts interpreted by the spatial correlation model. Improving the spatial correlation model and eliminating nonadmissible 
assumptions, finding perhaps a synthesis between both models. Elaborating the mathematical form of the model.

(3) Endeavouring to explain further empirical details by the spatial correlation model and extension of the knowledge on electron correlations in crystals. Extension of the existing interpretations to other metallic and nonmetallic structures. 